\title{
Quantitative Characterization of Protein Nanostructures Using Atomic Force Microscopy
}

\author{
Ine Segers-Nolten, Kees van der Werf, Martijn van Raaij, Vinod Subramaniam, Member, IEEE
}

\begin{abstract}
The aggregation of proteins into fibrillar structures called amyloid is a characteristic of many diseases, including several neurodegenerative disorders. Although amyloid formation is inherent to several serious diseases, the mechanism of fibril formation and the modes of toxicity are not yet known. High concentrations of fibrillar aggregates of $\alpha$ synuclein protein are found in the brains of patients suffering from Parkinson's disease. We exploit different contrast modes of high resolution atomic force microscopy (AFM) on fibrils formed by the wild-type $\alpha$-synuclein protein, and by the familial disease-related A30P, E46K and A53T variants, to get more insight into the in vitro process of fibril assembly. From quantitative analysis of height images measured in tapping mode AFM, we obtained data that are compatible with a twisted hierarchical assembly model [1] for all protein variants. The E46K mutant displays the most distinct and smallest periodicity. The modulation depth for all mutants is very similar, and is smaller for wild-type protein commensurate with the lower fibril height. The detailed morphology observed in phase images indicates however that fibrils may also be formed through the association of fibril segments. To study the mechanical properties of fibrils we applied force while scanning in contact mode, resulting in characteristic deformation of protein fibrils with a periodicity corresponding to the modulation observed in tapping mode. Our observations suggest that the hierarchical assembly model may not be the exclusive mechanism of $\alpha$-synuclein fibril assembly, but that multiple modes of fibril assembly play a role in $\alpha$-synuclein fibril formation.
\end{abstract}

\section{INTRODUCTION}

Several frequently occurring human diseases are accompanied by the accumulation of fibrillar protein aggregates, or amyloid. The aggregation of different proteins is characteristic of Alzheimer's disease, Huntington's disease, Creutzfeldt-Jacob disease, and Parkinson's disease [2]. In Parkinson's disease, fibrillar aggregates of $\alpha$-synuclein protein are found in inclusions in the brain called Lewy bodies. A relation between the

Manuscript received June 22, 2007. This work was supported in part by the research programme of the Stichting voor Fundamenteel Onderzoek der Materie, which is financially supported by the Nederlandse Organisatie voor Wetenschappelijk Onderzoek.

All authors are with the Biophysical Engineering Group, MESA+ Institute for Nanotechnology, University of Twente, PO Box 217, $7500 \mathrm{AE}$ Enschede, the Netherlands.

I. Segers-Nolten (phone: +31-53-4893726; fax: +31-53-4891105; email: g.m.j.segers-nolten@utwente.nl).

K. O. van der Werf (e-mail: k.o.vanderwerf@utwente.nl).

M. E. van Raaij (e-mail: m.e.vanraaij@utwente.nl).

V. Subramaniam (phone: +31-53-4893158; fax: +31-53-4891105; email: v.subramaniam@utwente.nl). aggregation of $\alpha$-synuclein and development of the disease has been proposed [3]. There is however no consensus yet on what species, formed during the aggregation process, is responsible for the degradation of neuronal cells. There are indications that early aggregation intermediates or small oligomeric structures are more toxic to cells than mature fibrils [4,5]. $\alpha$-Synuclein is an intrinsically unstructured protein and does not contain significant secondary structure in its native form. The structural flexibility mediates the capability to interact with many different types of binding partners [6]. Although the exact function of $\alpha$-synuclein in the human body is not yet clear, a chaperone function has been proposed, as has involvement in vesicle trafficking $[7,8]$. $\alpha$-Synuclein can fold into an $\alpha$-helix conformation upon binding to phospholipid membranes or vesicles and form $\beta$-sheet structures during aggregation into fibrils $[9,10]$. It is important to resolve the mechanisms of fibril formation, and the details of the potential pathogenic role of the fibrillar structures. Detailed insights into the process of aggregation are expected to offer possibilities to develop strategies to intervene in the progression of the disease. The dominant model for amyloid fibril assembly in the literature is the hierarchical assembly model postulating the initial formation of thin protofilaments and their subsequent twisting into protofibrils and mature fibrils [1]. An alternative model for insulin amyloid formation describes the assembly of fibril segments in a multi-pathway assembly mechanism [11]. We have studied $\alpha$-synuclein aggregation mechanisms in detail using the different contrast modes of high resolution atomic force microscopy on fibril samples prepared in vitro from recombinantly produced wild-type human $\alpha$-synuclein and the familial disease-related A30P, E46K and A53T mutant proteins. Detailed analyses of the AFM data yield quantitative information on the ultrastructural characteristics of $\alpha$-synuclein fibrils. Based on these results we propose that $\alpha$-synuclein aggregation may take place according to a multi-pathway mechanism.

\section{Materials AND MethodS}

\section{A. Expression and purification of $\alpha$-synuclein}

Recombinant expression and purification of human wildtype and A30P, E46K and A53T disease mutant $\alpha$-synuclein protein was performed as described previously [12].

\section{B. Aggregation reactions}

Monomeric $\alpha$-synuclein variants were incubated at a 
concentration of $100 \mu \mathrm{M}$ at $37{ }^{\circ} \mathrm{C}$ in $10 \mathrm{mM}$ Tris- $\mathrm{HCl}, 50$ $\mathrm{mM} \mathrm{NaCl}, \mathrm{pH} 7.4$ in glass vials under constant shaking. A Thioflavin $\mathrm{T}$ fluorescence assay was used to monitor the aggregation state. Samples for AFM measurement were taken when the Thioflavin $\mathrm{T}$ fluorescence had just reached its maximum intensity. For details see ref. [12].

\section{Atomic Force Microscopy imaging}

Atomic force microscopy images were made on a custom built instrument [13] using $\mathrm{Si}_{3} \mathrm{~N}_{4}$ tips (Veeco Instruments, Woodbury NY, USA, type MSCT-AUHW) with a spring constant of $0.5 \mathrm{~N} / \mathrm{m}$ and a nominal tip radius of $10 \mathrm{~nm}$. Imaging was performed in tapping mode in air at low force settings to minimize the interaction with the sample [14]. Both height and phase images were recorded.

Samples for AFM were prepared by applying $4 \mu \mathrm{l}$ of five times diluted protein sample on freshly cleaved mica. After 2 minutes of adsorption the sample was washed gently two times with $50 \mu \mathrm{l}$ MilliQ water and dried carefully under a stream of nitrogen.

Quantitative characterization of AFM height images was performed using SPIP software (Image Metrology A/S, Lyngby, Denmark) according to the procedure described in ref. [12].

\section{Mechanical fibril deformation measurements using Atomic Force Microscopy}

We alternated between tapping and contact mode scanning to study the mechanical properties of $\alpha$-synuclein fibrils. A part of the fibril sample was imaged at low interaction force in tapping mode, followed by contact mode scanning of the same area at a typical contact force of 15-20 $\mathrm{nN}$. Finally, a larger tapping mode image was generated to visualize the effect of force on the morphology of the fibrils.

\section{RESULTS}

\section{A. $\alpha$-Synuclein variants display different nanoscale fibril morphologies}

Aggregation half-times measured using Thioflavin $\mathrm{T}$ fluorescence spectroscopy at low salt reaction conditions indicated different aggregation kinetics for the variants: 150 hrs for wild-type and $75 \mathrm{hrs}, 100 \mathrm{hrs}$ and $250 \mathrm{hrs}$ for A53T, E46K and A30P respectively. These results are consistent with the literature $[15,16]$. AFM images in air (Fig. 1) were recorded to verify if the different aggregation kinetics influence the fibril morphologies. Visual inspection of the images suggests that the morphology is not identical for all variants. A quantitative analysis of heights, modulation depths, and periodicities of a large number of fibrils of each variant was performed. The resulting data (Table I) reveal that a single point mutation in the $\alpha$-synuclein protein has significant consequences for the ultrastructure of the fibrils formed.
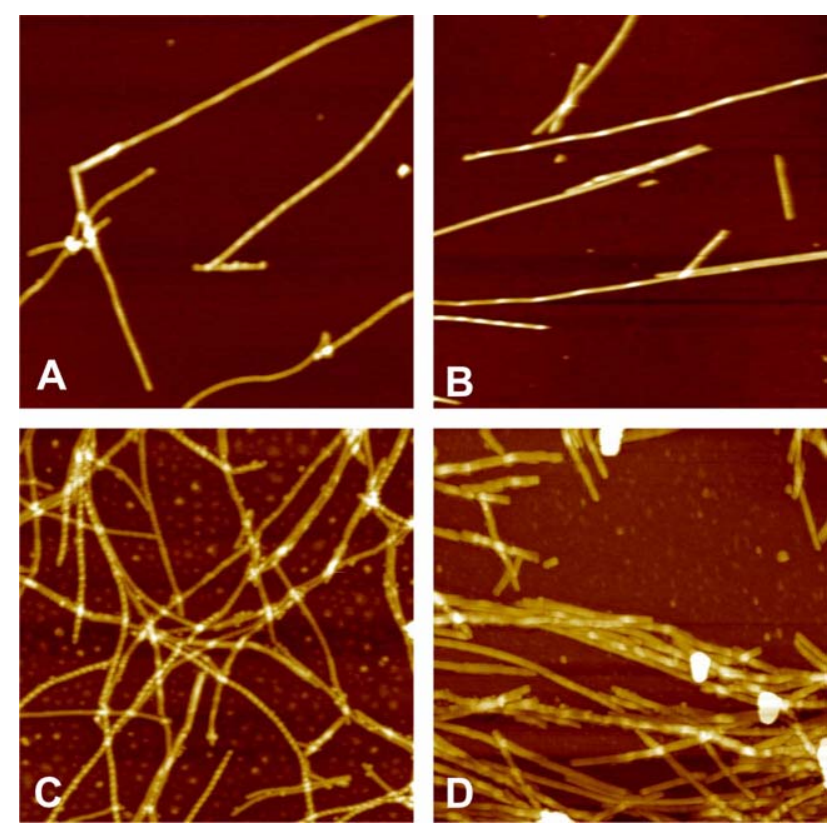

Fig.1. Representative tapping mode AFM images of fibrils of wild-type protein (A), and A30P (B), E46K (C) and A53T (D) variants of $\alpha$-synuclein in air. The color scale represents $15.6 \mathrm{~nm}$ in height for wild-type, $20.2 \mathrm{~nm}$ for $\mathrm{A} 30 \mathrm{P}, 22.4 \mathrm{~nm}$ for $\mathrm{E} 46 \mathrm{~K}$ and $31.3 \mathrm{~nm}$ for A53T $\alpha$-synuclein. Image size is $2500 \times 2500 \mathrm{~nm}$.

Wild-type fibrils exhibit the lowest height; A30P and E46K have intermediate values, while A53T fibrils are highest. The modulation depth is not significantly different among the mutant proteins, although wild-type protein has a smaller modulation depth commensurate with the lower measured fibril height. Together with the fibril height the periodicity of the fibrils is the most characteristic parameter for each $\alpha$-synuclein variant. The E46K mutant displays a very distinct average periodicity of about $60 \mathrm{~nm}, \mathrm{~A} 53 \mathrm{~T}$ an intermediate value of $110 \mathrm{~nm}$, and wild-type and A30P the largest periodicities of $\sim 140 \mathrm{~nm}$.

TABLE I. QUANTITATIVE CHARACTERISTICS OF FIBRILS OF ALPHA-SYNUCLEIN VARIANTS

\begin{tabular}{ccccc}
\hline variant & Nfibrils & $\begin{array}{c}\text { fibril top } \\
\text { height }(\mathrm{nm})\end{array}$ & $\begin{array}{c}\text { modulation } \\
\text { depth }(\mathrm{nm})\end{array}$ & $\begin{array}{c}\text { periodicity } \\
(\mathrm{nm})\end{array}$ \\
\hline wild-type & $33(27)$ & $7.5 \pm 0.9$ & $1.5 \pm 0.4$ & $141 \pm 82$ \\
A30P & $41(21)$ & $8.7 \pm 1.4$ & $2.3 \pm 0.7$ & $139 \pm 46$ \\
E46K & $37(29)$ & $9.8 \pm 1.2$ & $2.1 \pm 0.6$ & $59 \pm 28$ \\
A53T & $33(20)$ & $10.4 \pm 1.3$ & $2.2 \pm 0.8$ & $115 \pm 41$ \\
\hline
\end{tabular}

$\mathrm{N}$ fibrils is the total number of fibrils analyzed per mutant. The number of periodic fibrils is in parentheses. The fibrils classified as non-periodic were irregular in height. Height values are averages over all $N$ fibrils, while the modulation depth and periodicity are calculated for only the periodic fibrils. The standard deviation is used as the measurement error.

The distribution of period lengths is narrowest for E46K (main mode at 40-50 nm), whereas A30P and A53T display wider distributions. Wild-type protein displays a main periodicity of around $80 \mathrm{~nm}$, but larger periodicities were also observed (Fig. 2). The fibril heights and modulation depths measured in air for $\mathrm{A} 30 \mathrm{P}$ and E46K variants are consistent, within the accuracy of the data, with earlier AFM 
results in liquid [12]. The measured heights and modulation depths of wild-type protein are somewhat smaller in air than in liquid. Periodicities measured for A30P and E46K are similar in air and in liquid, and also for wild-type protein considering the main mode in the broad distribution. Quantitative AFM data in solution are not yet available for the A53T mutant.
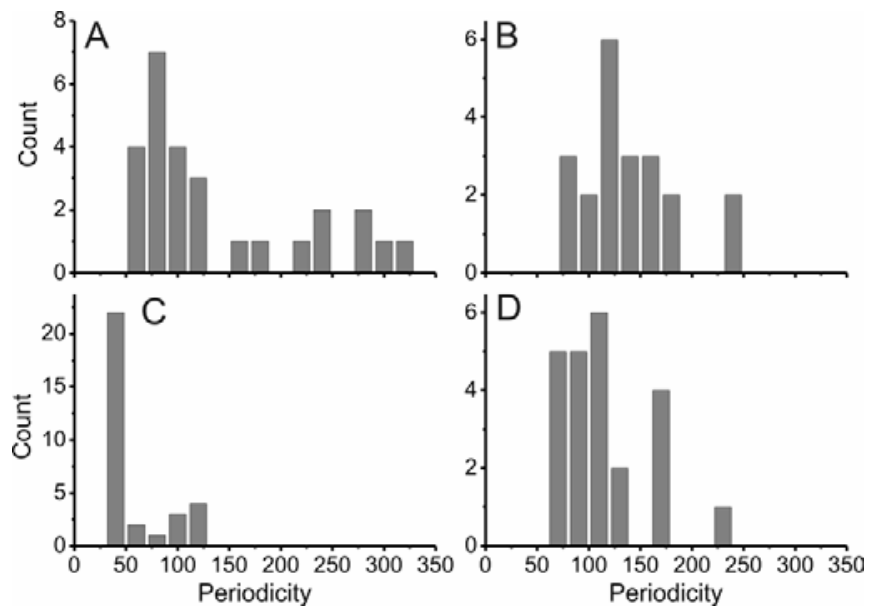

Fig.2. Distribution of fibril periodicities for wild-type (A), A30P (B), E46K (C) and A53T (D) variants of $\alpha$-synuclein. The number of fibrils analyzed is indicated in Table I. Histogram bin size is $20 \mathrm{~nm}$.

\section{B. Twisted versus segmented $\alpha$-synuclein fibril morphology}

High resolution phase images were recorded to examine the nanoscale structure of individual $\alpha$-synuclein fibrils in detail. Phase imaging is sensitive to differences in material properties like sample viscoelasticity or hydrophobicity, and is a complementary contrast mode yielding more detail than can be observed in height images [17].

Fibrils shown in Fig. 3A clearly display twisted fibril morphology, providing evidence for the presence of a skewed pitch. In contrast, Fig. 3B shows a high resolution image of a small area scan revealing assembly of fibril segments of 100-150 nm in length. Segmented morphologies are predominantly observed in lower height fibrils, attributed to be protofibrillar structures on the fibrillization pathway. Fig. 3C and 3D demonstrate that both assembly modes can be found within one sample, an observation made for all $\alpha$-synuclein variants.

\section{Mechanical deformation corroborates periodic morphology of $\alpha$-synuclein fibrils}

Tapping mode AFM is most suited for imaging soft biological samples [1], and minimal tip-sample interactions maintain the integrity of the sample. Fig. 4A depicts an image of $\mathrm{A} 30 \mathrm{P}$ mutant fibrils obtained by scanning in tapping mode. The same area was then rescanned in contact mode, followed by another larger scan in tapping mode. Fig. 4B combines the images taken before and after contact mode scanning, and clearly shows deformed fibrils in the area scanned in contact mode; outside this area the fibrils are not affected. The regular pattern of nanoscale deformations is the result of the force imposed on the fibrils during contact mode scanning. A closer look at a single fibril reveals that the spacing between the deformations corresponds to the $140 \mathrm{~nm}$ periodicity of the original fibrils (Fig. 4C, D and E).
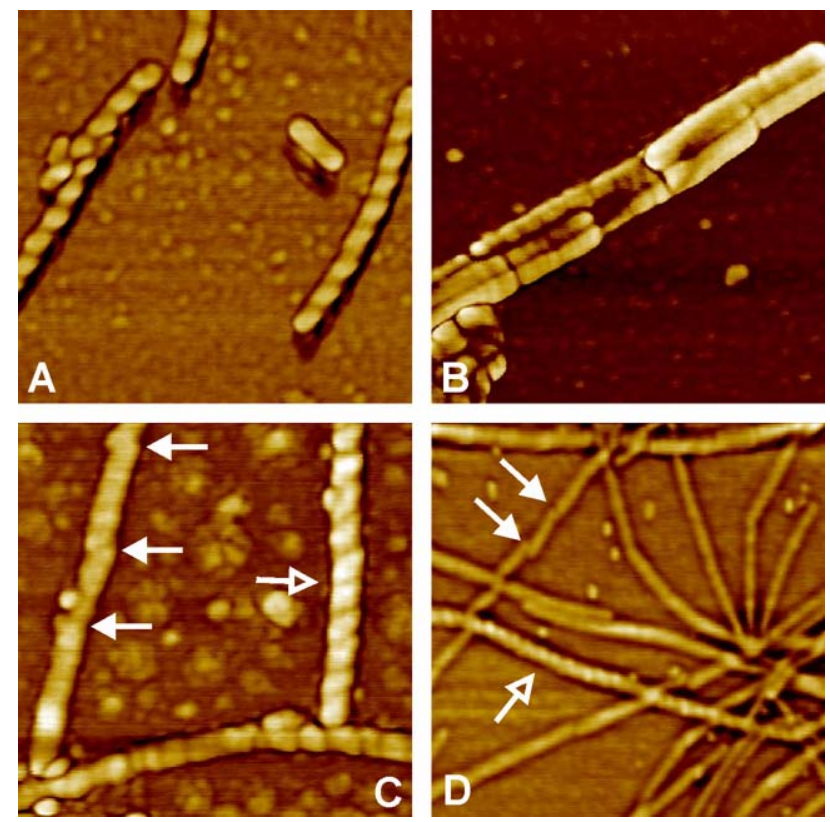

Fig.3. Tapping mode AFM phase images in air. (A) E46K fibrils displaying twisted morphology. (B) Wild-type fibril with appearance of associated segments. (C) and (D) contain both twisted and segmented E46K fibrils. Image sizes : A, B and C, $620 \times 620 \mathrm{~nm}$; D, $1250 \times 1250 \mathrm{~nm}$. Closed arrows point to segmented fibrils, open arrows to twisted fibrils.

\section{DISCUSSION}

There is substantial evidence that $\alpha$-synuclein plays a pathogenic role in Parkinson's disease. Lewy bodies containing high concentrations of fibrillar $\alpha$-synuclein aggregates [3] are found in post-mortem brain tissues of Parkinson's patients suffering from both sporadic and familial forms of the disease. Transfection with human $\alpha-$ synuclein in animal models results in the development of $\alpha$ synuclein containing inclusion bodies in combination with the appearance of typical symptoms like motor control loss [18]. Apparently $\alpha$-synuclein has a normal, still unknown, function at low concentrations, but potentially may transform into a pathogenic species at high concentration. Specific point mutations in the $\alpha$-synuclein gene inevitably result in early-onset Parkinson's disease. These specific mutations have an effect on the detailed morphological appearance of $\alpha$-synuclein fibrils formed in vitro, as shown in Fig.1. High-resolution AFM at low forces enables the non-invasive monitoring of structure and formation of biological nanostructures, yielding insights into the underlying aggregation mechanisms. The ability to operate in solution enables a detailed exploration by AFM of the fibrillization phase space as a function of time and solution 
conditions. In this work we have focused on exploiting different AFM contrast modes in air to reveal quantitative details about nanometer scale fibril morphologies.

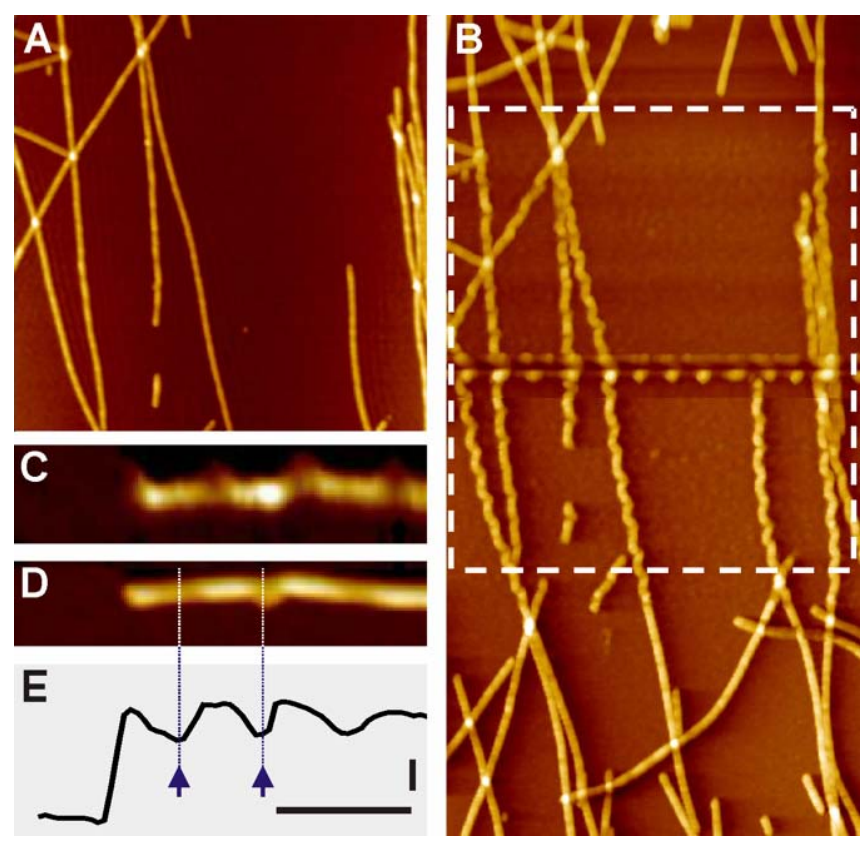

Fig. 4. (A) Tapping mode AFM image of A30P $\alpha$-synuclein fibrils taken before nanomanipulation. Image size is $2500 \times 2500 \mathrm{~nm}$. (B) Composite image of two images taken in tapping mode, before and after the deformation in contact mode. The horizontal track in the middle of the contact mode area indicated by the dashed box is the result of repeated scanning during the procedure for optimization of scan parameters and settings. The tip becomes less sharp because of the experiments in contact mode, and due to tip convolution the fibrils appear wider than in Fig. 4A. Image size is $2700 \times 5400 \mathrm{~nm}$. (C) and (D) Close up of a single fibril before (D) and after deformation (C), the arrows indicate an identical periodicity in both fibrils of $\sim 140 \mathrm{~nm}$ (E). In (E) the horizontal scale bar is $225 \mathrm{~nm}$, the vertical scale bar is $2 \mathrm{~nm}$.

Our results clearly indicated that the aggregation of genetic variants of $\alpha$-synuclein not only follows different kinetics but is also accompanied by the formation of morphologically different fibrils. Individual mutant forms of $\alpha$-synuclein fibrils may be distinguished on the basis of their characteristic heights and periodicities. Specific point mutations appear to affect fibril assembly and may drive fibril formation into energetically more favorable structures with characteristic periodicity. The measured modulation depths in relation to the fibril heights are compatible with a twisted hierarchical assembly model within the accuracy of the data. For this model we expect the modulation depth not to exceed $25 \%$ of the maximum height of the fibril, an expectation supported by the data shown in Table I. However, we also observe morphologies that do not resemble the expected twisted structure, but appear like a series of associated segments. This morphology has also been observed for insulin fibrils [11]. We thus have evidence for both hierarchical and segmented $\alpha$-synuclein fibril formation, potentially based on a multi-pathway mechanism. We speculate that segmented assembly may precede the twist formation, because we mainly observe segmented morphologies in lower height fibrils attributed to protofibrillar structures. Higher resolution imaging and further analysis is required for definitive conclusions.

The contact mode experiments resulted in fibril deformations spaced at distances identical to the periodicity of the intact fibrils. Lateral forces in contact mode are much higher than in tapping mode because the tip is continuously in contact with the sample. The contact force is mainly dominated by the adhesion force between the $\mathrm{Si}_{3} \mathrm{~N}_{4}$ tip and the sample. The periodicity of the deformations may arise from the characteristic fibril structure, assembled in such a way that it is locally more sensitive to deformation by lateral force. Another reason may be the intrinsic variation in adhesion force. Hydrophilic parts of the sample will display higher adhesion forces [19], and may be more prone to damage by high local lateral forces. The fibril deformation results are also consistent with both hierarchical and segmented $\alpha$-synuclein fibril assembly. Further high resolution imaging of these protein nanostructures using low-force imaging and the sharpest AFM tips available are in progress. In combination with well-characterized tipsample interactions, these approaches are expected to yield definitive insights into fibril assembly mechanisms.

\section{ACKNOWLEDGMENT}

The authors thank Kirsten van Leijenhorst-Groener for protein expression and purification.

\section{REFERENCES}

[1] R. Khurana, et al., Biophys. J., vol. 85, pp. 1135-1144, 2003

[2] F. Chiti, and C.M. Dobson, Ann. Rev. Biochem., vol. 75, pp. 333-366, 2006

[3] M.G. Spillantini, et al., Nature, vol. 388, pp. 839-840, 1997

[4] H.A. Lashuel, et al., J. Mol. Biol., vol. 322, pp. 1089-1102, 2002

[5] T.T. Ding, S.J. Lee, J.C. Rochet and P.T. Lansbury Jr., Biochemistry, vol. 41, pp. 10209-10217, 2002

[6] V.N. Uversky, Eur. J. Biochem., vol. 269, pp. 2-12, 2002

[7] J.M. Souza, B.I. Giasson, V.M. Lee, and H. Ischiropoulos, FEBS Lett., vol. 474, pp. 116-119, 2000

[8] D.D. Murphy, S.M. Rueter, J.Q. Trojanowski and V.M. Lee, J. Neurosci., vol. 20, pp. 3214-3220, 2000

[9] W.S Davidson, A. Jonas, D.F. Clayton and J.M. George, J. Biol. Chem., vol. 273, pp. 9443-9449, 1998

[10] L.C. Serpell, J. Berriman, R. Jakes, M. Goedert and R.A. Crowther, Proc. Natl. Acad. Sci. USA, vol. 97, pp.4897-4902, 2000

[11] R. Jansen, W. Dzwolak and R. Winter, Biophys J., vol. 88, pp. 1344 1353,2005

[12] M.E. van Raaij, I.M. Segers-Nolten and V. Subramaniam, Biophys J., vol. 91, L96-98, 2006

[13] K.O. van der Werf, et al., Rev. Sci. Instr., vol. 64, pp. 2892-2897, 1993

[14] H.G. Hansma, et al., Scanning, vol. 15, pp. 296-299, 1993

[15] W. Hoyer, D. Cherny, V. Subramaniam and T.M. Jovin, Biochemistry, vol. 43, pp. 16233-16242, 2004

[16] R.A. Fredenburg et al., Biochemistry, vol. 46, pp. 7107-7118, 2007

[17] M. Argaman, R. Golan, N.H. Thomson and H.G. Hansma, Nucleic Acids Res., vol. 25, pp. 4379-4384, 1997

[18] B.I. Giasson, et al., Neuron., vol. 34, pp. 521-533, 2002

[19] S.J.T. van Noort, K.O. van der Werf, B.G. de Grooth, N.F. van Hulst, and J. Greve, Ultramicroscopy, vol. 69, pp. 117-127, 1997 